\title{
Combining in-Situ SEM with High Sensitivity Analytical TEM for Understanding the Degradation of Metallic Interconnects in SOFC
}

\author{
Stéphane Poitel ${ }^{1,3}$, Zhu-Jun Wang ${ }^{2}$, Marc Willinger ${ }^{2}$, Jan van Herle $^{3}$ and Cécile Hébert ${ }^{1}$ \\ 1. Centre Interdisciplinaire de Microscopie Electronique, Ecole Polytechnique Fédérale de Lausanne, \\ Lausanne, Switzerland. \\ 2. Department of Inorganic Chemistry, Fritz Haber Institute of the Max Planck Society, Berlin, Germany. \\ 3. Group of Energy Materials, Ecole Polytechnique Fédérale de Lausanne, Sion, Switzerland.
}

Solid Oxide Fuel Cells (SOFC) are very effective energy converters. They allow obtaining electricity and heat from fuels like natural gas or hydrogen and will play a key role in the energy transition.

Among the critical components of SOFC, interconnects are coated steel plates. Their main roles are to collect current, separate the gases and to ensure mechanical stability of the stack. The main problem is that at $700-800^{\circ} \mathrm{C}$ (SOFC working temperature), the chromium present in the steel to prevent its oxidation tends to evaporate and contaminate other parts of the stack [1]. Hence coatings are necessary to protect chromium from evaporation. Several SOFC metallic interconnect coatings are based on cobalt, which is efficient to prevent chromium evaporation and to maintain the contact resistance low after prolonged exposure [2]. The addition of a very thin cerium layer beneath the Co coating seems to reinforce its properties [3]. The oxidation of such coated SOFC FeCr steels, which contain a considerable number of alloy additives, becomes difficult to interpret.

Getting insight into the degradation mechanisms of interconnects is crucial to be able to design reliable elements that will perform over the lifetime of the SOFC. Here we report on the combined use of in-situ scanning electron microscopy in an in-house modified FEI Quanta 200 environmental SEM (ESEM) and post mortem analytical TEM of sample extracted by FIB from the in-situ oxidized specimens.

The sample was a Sanergy SSHT steel with Ce-Co coating, frequently used as interconnect material. It was observed at high temperature $\left(900^{\circ} \mathrm{C}\right)$ under an oxygen flow, in ESEM, for durations from 0 to60.

In order to be close to operation conditions, one should heat the sample under vacuum to $900^{\circ} \mathrm{C}$ and then introduce oxygen and record images. Due to the gas introduction at high temperature (and hence switch from high vacuum mode to ESEM mode), the beginning of the reaction is challenging to record. To record the beginning of the experiment, it was tried to heat at slower rate, heat under hydrogen and introducing the oxygen at a slower rate but all these modifications induce different final morphology.

In-situ observation of the oxidation shows grain growth and coarsening for the first 10 hours. Then a change in morphology appears. The symmetry of the crystal seems to evolve from cubic to another structure.

TEM lamellae are then extracted from the in-situ observed areas using a focused ion beam (FIB). This allowed to evaluate the final composition of the oxidation layers of the steel by scanning transmission electron microscopy (STEM) and energy dispersive x-ray analysis (EDX) (figure 2). 
We observe a complex migration of species, especially, the diffusion of manganese towards the cobalt coating to form a cobalt-manganese spinel oxide layer. Under this top layer, chromia scale forms. The very thin cerium layer does not prevent the diffusion of other elements from the steel. Niobium and titanium form oxidized precipitates in the steel just below the chromia scale.

Results from shorter exposure/observation times present a time-lapse picture of the initial oxidation for a more detailed understanding. Ex-situ experiments complement the results.

\section{References:}

[1] Fergus, J. W, International Journal of Hydrogen Energy 32 (2007), p. 3664.

[2] Froitzheim, J. \& Svensson, J.-E., ECS Trans. 35 (2011), p. 2503.

[3] Harthøj, A., Holt, T. \& Møller, P. Journal of Power Sources 281 (2015), p. 227.
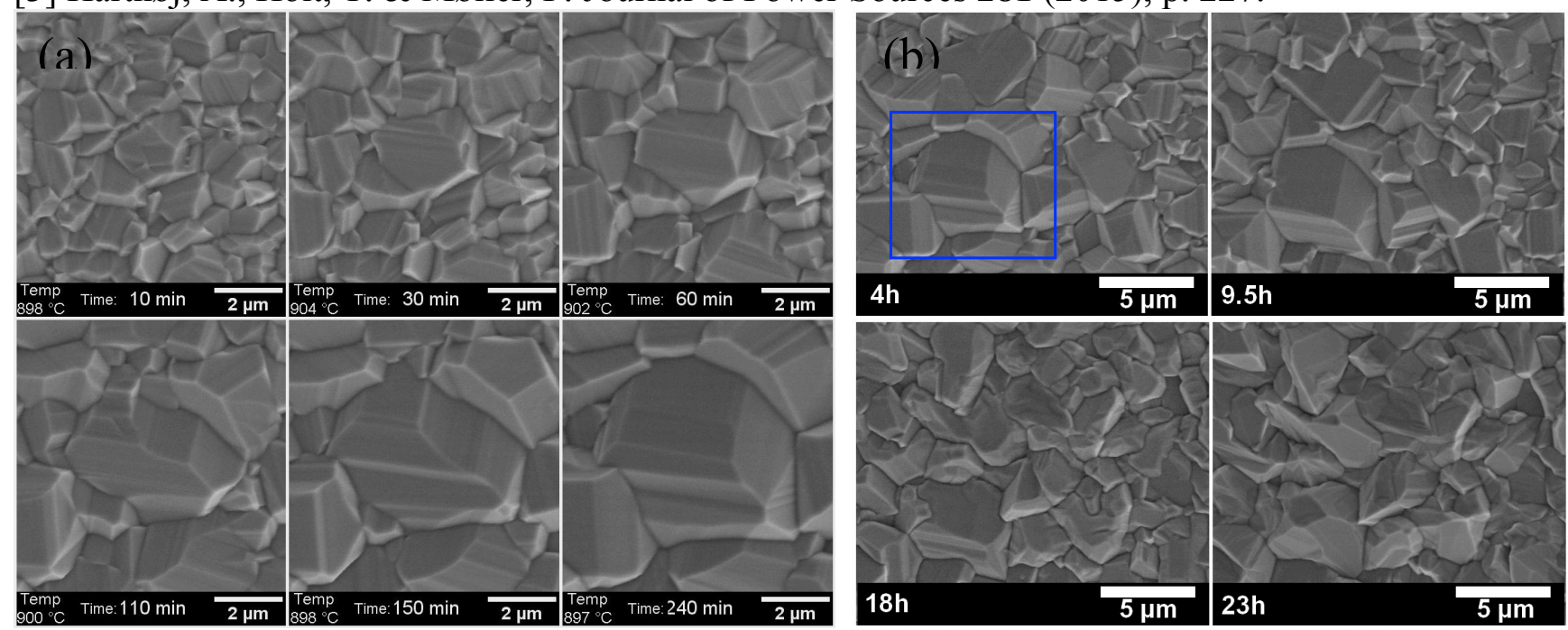

Figure 1. (a) grain growth at the surface of the sample at $900^{\circ} \mathrm{C}$ under $30 \mathrm{~Pa}$ of $\mathrm{O}_{2}$. (b) Morphology change after 4-23h exposure to same condition (blue rectangle highlight the grain that growth is showed on (a)).

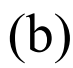

\section{(a)}
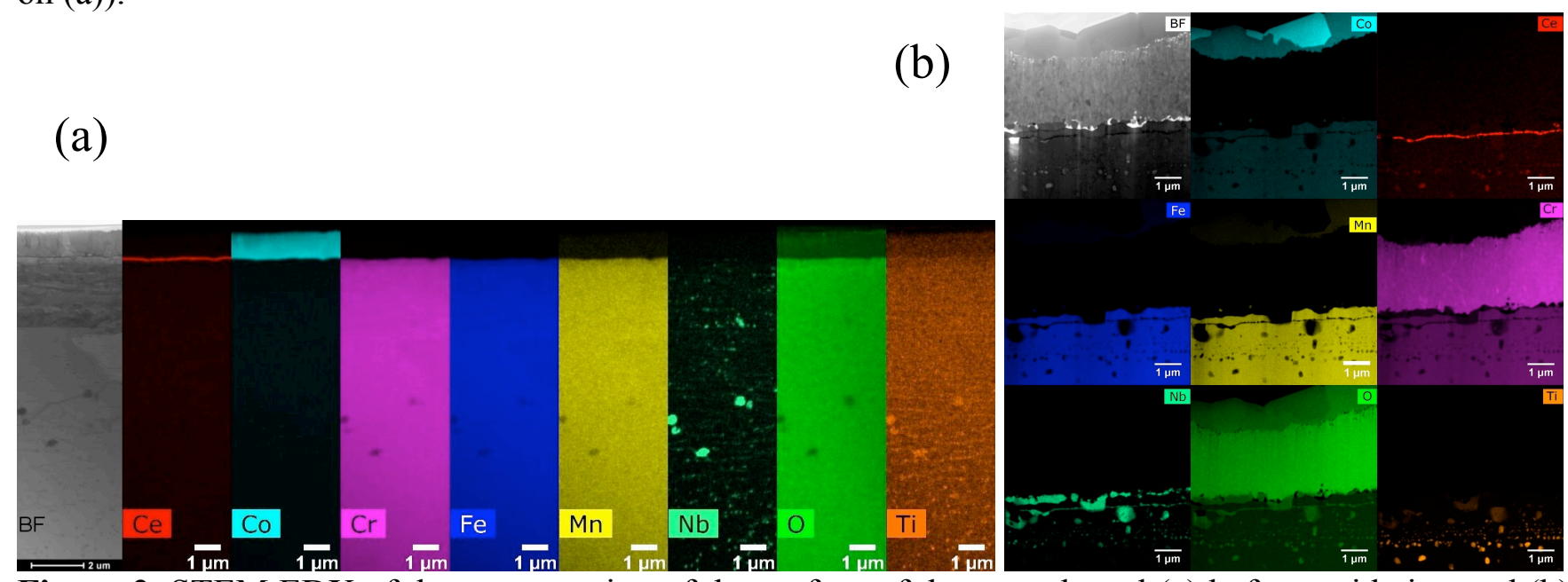

Figure 2. STEM EDX of the cross section of the surface of the coated steel (a) before oxidation and (b) after 48 hours oxidation at $900^{\circ} \mathrm{C}$ under $30 \mathrm{~Pa} \mathrm{O}_{2}$. 